\title{
Refractory Ovarian Carcinoma
}

National Cancer Institute

\section{Source}

National Cancer Institute. Refractory Ovarian Carcinoma. NCI Thesaurus. Code C150091.

Ovarian carcinoma that is resistant to treatment. 